\title{
Environmental problems of sustainable development of oil-producing regions of the North
}

\author{
Aigul Yamaletdinova ${ }^{1}$, Viktor Barkhatov ${ }^{2}$, Sergey Gots $^{2}$, Klara Yamaletdinova ${ }^{2, *}$, Azamat \\ Nurutdinov $^{2,3}$, and Marat Tuhvatullin ${ }^{1}$ \\ ${ }^{1}$ Ufa State Petroleum Technological University, 1, Kosmonavtov str., 450062, Ufa, Russia \\ ${ }^{2}$ Chelyabinsk State University, 129, Bratiev Kashirinykh str., 454001 Chelyabinsk, Russia \\ ${ }^{3}$ Bashkir State University, 32, Zaki Validi str., 450076, Ufa, Russia
}

\begin{abstract}
There is a relationship between the economic requirements for the development of oil and gas facilities in the oil-producing regions of the North and the environmental problems of sustainable development. One of the ways to solve this problem is the wider use of underground gas storage facilities both for reducing peak loads in the Unified Gas Supply System by collecting and storing previously flared associated petroleum gas, and for burying a large volume of industrial emissions that are harmful to the environment. We have considered a mathematical model of an automated control system for the creation and operation of underground gas storage in the horizons of a depleted oil and gas condensate field with its subsequent use for disposal of industrial emissions. To ensure the stability of the functioning of objects of accumulation and consumption, it is necessary to calculate the depth of feedback for each component of the component: proportional, integral, differential.
\end{abstract}

\section{Introduction}

The world community has long been concerned about the solution to the issue of achieving an ecological balance in the environment. To this end, the Kyoto Protocol was signed - an international agreement in the UN Framework Convention, according to which each industrialized state received certain quotas for the mass of emissions of harmful gases into the atmosphere, which would help prevent anthropogenic impact on the planet's climate system. The Paris Agreement on Climate, adopted instead of the Kyoto Protocol, regulating measures to reduce anthropogenic impact on the atmosphere from 2020, unfortunately, does not provide for a mechanism to enforce the obligation to achieve them.

In the modern paradigm of sustainable development of the oil and gas complex, environmental problems play an important role. The solution of environmental problems must be attributed primarily to the prevention of harm to the environment. The use of new technologies [4] contributes to the development of such important components of sustainable development as trust [8] and horizontal interactions [9]. Sustainable

\footnotetext{
*Corresponding author: clara-yk@yandex.ru
} 
development itself has a positive effect on the country's ability to withstand external challenges to be stronger in geopolitical and economic terms [10]

The anthropogenic impact on the atmosphere of the air environment also includes all the more frequent cases of accidental spills of oil and oil products. Due to the fact that the main large oil and gas producing fields are currently located in the Far North, it is very important for the sustainable development of oil producing regions of the North to develop measures to prevent and eliminate spills of oil and oil products. These measures consist in strict observance of all the requirements of the Regulations for the exploration, development, production, collection and transportation of gas, oil and oil products by both large companies and a considerable number of small companies, with the obligatory development, implementation and responsible fulfillment of all technological requirements in accordance with ISO 9001:2015, ISO 14001:2015 and ISO 45001:2018. At the same time, the statistics of accidental oil and oil products spills show that the equipment and technologies for drilling and completing oil and oil and gas wells require further research and field tests. In addition, the task of developing a liquidation technology is very urgent so that abandoned fields and wells do not become sources of regional environmental disasters with all the consequences. Often accidents and environmental disasters are associated with savings on preventive measures when using products by external consumers.

\section{Materials and methods}

The environmental problems of sustainable development of oil-producing regions of the North today also depend on the economic requirements for the development of oil and gas facilities, in connection with which it is recommended to use underground gas storage facilities not only to reduce peak loads in the Unified Gas Supply System by collecting and storing previously flared associated petroleum gas, but also for the disposal of large volumes of industrial emissions that are harmful to the environment.

From this point of view, we will consider a mathematical model of an automated control system for the creation and operation of underground gas storage in the horizons of a depleted oil and gas condensate field with its subsequent use for disposal of industrial emissions.

\section{Results}

In a depleted oil and gas condensate field, where, together with the cyclic operation of an underground gas storage facility, residual condensate is also withdrawn, it is recommended to dispose of industrial emissions. At present, the Yareyuskoye oil and gas condensate field is being developed in the Far North region. Several oil and gas fields are being developed not far from this field, where the issue of utilizing unclaimed volumes of associated petroleum gas has not been resolved. This predetermines the relevance of the study and scientific substantiation of the creation of an underground gas storage both for associated petroleum gas and for the disposal of industrial emissions, based on a depleted gas condensate field on the example of the Yareyuskoye oil and gas condensate field, for example, carbon dioxide.

The fact of the existence of an oil and gas condensate field testifies to the tightness of the roof. In a purely ecological aspect, it is not so much the fact of the existence of the oil and gas condensate field that is important as the dynamics of changes in time $\mathrm{Y}_{\mathrm{Hi}}(\mathrm{t})$ of the volumes (proportional, integral, differential (PID) characteristics of the potentials) of the accumulation of its main components: oil $\mathrm{Y}_{\mathrm{H} 1}(\mathrm{t})$, gas (gases) $\mathrm{Y}_{\mathrm{H} 2}(\mathrm{t})$, gas condensate, $\mathrm{Y}_{\mathrm{H} 3}(\mathrm{t})$ ballast (water) $\mathrm{Y}_{\mathrm{H} 4}(\mathrm{t})$, solid $\mathrm{Y}_{\mathrm{H} 5}(\mathrm{t})$, liquid $\mathrm{Y}_{\mathrm{H} 6}(\mathrm{t})$, gaseous $\mathrm{Y}_{\mathrm{H} 7}(\mathrm{t})$ industrial waste. In 
addition to accumulation potentials, of great interest are the PID characteristics of the volumes of production (consumption) of oil $Z_{\mathrm{P} 1}(\mathrm{t})$, gas (gases) $\mathrm{Z}_{\mathrm{P} 2}(\mathrm{t})$, gas condensate, $\mathrm{Z}_{\mathrm{P} 3}(\mathrm{t})$ ballast (water) $\mathrm{Z}_{\mathrm{P} 4}(\mathrm{t})$, solid $\mathrm{Z}_{\mathrm{P} 5}(\mathrm{t})$, liquid $\mathrm{Z}_{\mathrm{P} 6}(\mathrm{t})$, gaseous $\mathrm{Z}_{\mathrm{P} 7}(\mathrm{t})$ industrial waste. In a more familiar aspect, these characteristics determine the change in pressure and flow rates in wells over time, the geological and physical parameters of the reservoir, and the physical properties of condensate, gas and water. At the same time, studies are being carried out to determine the future flow rates of such wells, the operating mode of the UGS facility, the maximum possible volume of residual condensate recovery, measures to increase the productivity of injection-producing wells, and changes in the gas composition during underground storage [1].

However, it is necessary to carefully inspect, select and repair old abandoned or leaking wells, study the condition and tightness of loops, field oil pipelines, separators and other equipment for the possibility of their use in the process of underground gas storage, reconstruct field gas pipelines, build new installations for gas purification and dehydration, to drill new injection and production wells [2]. Despite the above difficulties, the costs of creating underground gas storage facilities, especially in the Far North far from the Unified Gas Supply System, are significantly lower than the costs of creating corresponding capacities for transport and disposal of substances that harm the environment.

Despite the fact that several of the noted problems are solved by strict compliance with the requirements of legislative and regulatory documents of the state, at the same time, in order to solve the problem of reducing peak loads in the Unified Gas Supply System by collecting and storing previously flared associated petroleum gas and for burying a large volume of carbon dioxide, causing harm to the environment, it is recommended to develop methods for regulating the sustainable operation of underground gas storage.

Sustainable operation of underground gas storage assumes five different options and stages of its operation:

1. Consumption $\mathrm{Y}_{\mathrm{P} 2}(\mathrm{t})$ of gas is proportional to its accumulation $\mathrm{Y}_{\mathrm{H} 2}(\mathrm{t})$, i.e. the volumes of injected gas $\mathrm{X}_{\mathrm{Z2}}(\mathrm{t})$ are equal to the volumes of the extracted gas;

2. The volumes $X_{32}(t)$ of the injected gas are greater than the volumes of $Y_{\Pi 2}(t)$ consumed. Over time, an overflow of an underground reservoir, a breach of the tightness of the rock and a spontaneous release of gas to the surface through the formed cracks (the socalled griffins) can occur;

3. The volumes $\mathrm{X}_{32}(\mathrm{t})$ of the injected gas are less than the volumes of $\mathrm{Y}_{\Pi 2}(\mathrm{t})$ consumed. Underground gas storage works for depletion. Gradually, the pressure drops below the saturation pressure of the condensate with gas, which will cause the condensate to drop out and settle on the walls of the formation pores;

4. A rim of accumulated saline water $\mathrm{Y}_{\mathrm{H} 3}(\mathrm{t})$ is injected, in which associated petroleum gas, gas condensate, and also carbon dioxide prepared for burial are slightly dissolved.

5. Following the rim of saline water, the volume $\mathrm{Y}_{\mathrm{H} 7}(\mathrm{t})$ of accumulated carbon dioxide, prepared for burial, decreases.

To analyze the sustainability of the underground gas storage facility, it is advisable to consider various control methods (Figure 1):

- at the entrance (download);

- on exit (selection);

- by feedback.

Let us consider a mathematical model of the operation of an underground gas storage, taking into account the risk of instability and instability in the control of the process of accumulation of hydrocarbon mixtures and carbon dioxide in UGS facilities with preliminary recirculation of hydrocarbon mixtures, where the accumulation of carbon dioxide is considered without recirculation. 


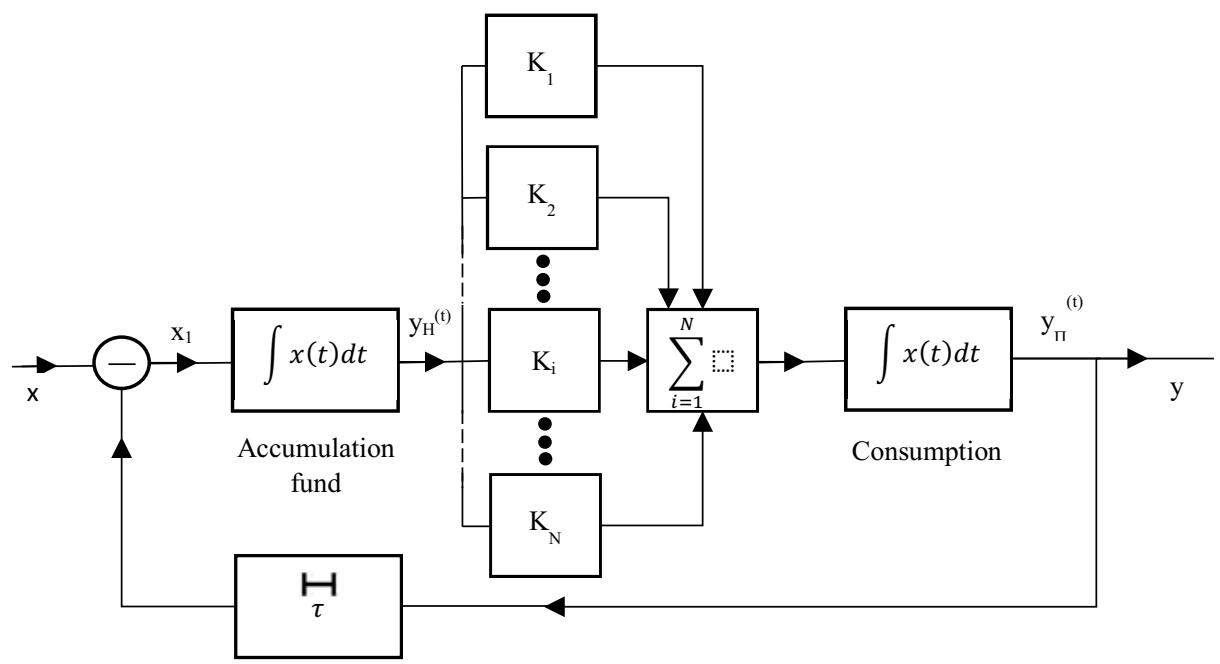

Fig. 1. Functional diagram of accumulation and consumption control in the parameter stabilization mode with negative feedback.

Figure 1 shows a functional diagram of accumulation and consumption control in the mode of stabilization of parameters with negative feedback for various options and stages of operation of an underground gas storage facility based on a depleted oil and gas condensate field.

The amount of accumulated production in the form of associated petroleum gas, mineralized water, carbon dioxide and gas condensate $\mathrm{y}_{\mathrm{H}}(\mathrm{t})$ in the accumulation fund is determined by the formula:

$$
y_{H}(t)=\frac{1}{T_{H}} \int_{0}^{t} X_{1}(\theta) \cdot d \theta
$$

where $\mathrm{T}_{\mathrm{H}}$ is the accumulation time constant. $\mathrm{T}$ significantly exceeds the time of the full cycle of recirculation of the injected associated gas into the UGS facility. The amount of consumed products in the form of associated petroleum gas and gas condensate from the accumulation fund is found by the formula:

$$
y_{\Pi}(t)=\frac{1}{T_{\Pi}} \int_{0}^{t} \cdot\left[\sum_{i=1}^{N} K_{i}(t)\right] \cdot y_{H}(t) \cdot d t
$$

$\mathrm{T}_{\mathrm{P}}$ is the time constant of consumption and injection of carbon dioxide, $\mathrm{K}_{\mathrm{i}}$ is the volume of the planned $\mathrm{i}$-th consumption of gas and gas condensate. The total produced production from the reservoir, consisting of injected associated petroleum gas and gas condensate, which should not exceed one.

$$
\sum_{i=1}^{N} K_{i}(t) \leq 1
$$

Substituting $\mathrm{y}_{\mathrm{H}}(\mathrm{t})$ from (1) into (2), we have 


$$
y_{\Pi}(t)=\frac{1}{T_{\Pi} \cdot T_{H}} \int_{0}^{t} \cdot\left[\sum_{i=1}^{N} K_{i}(t)\right] \cdot \int_{0}^{t} X_{1}(\theta) \cdot d \theta \cdot d t
$$

The performed integration operations are equivalent to averaging the parameters over time. Therefore (4) can be rewritten as follows

$$
y_{\Pi I}(t)=\overline{x_{1}(\theta) \times \overline{\left[\sum_{i=1}^{N} K_{i}(t)\right]}} .
$$

Formulas (1) and (5) make it possible to control the operation of underground gas storage by regulating its parameters at various options and stages of its operation.

feedback regulation

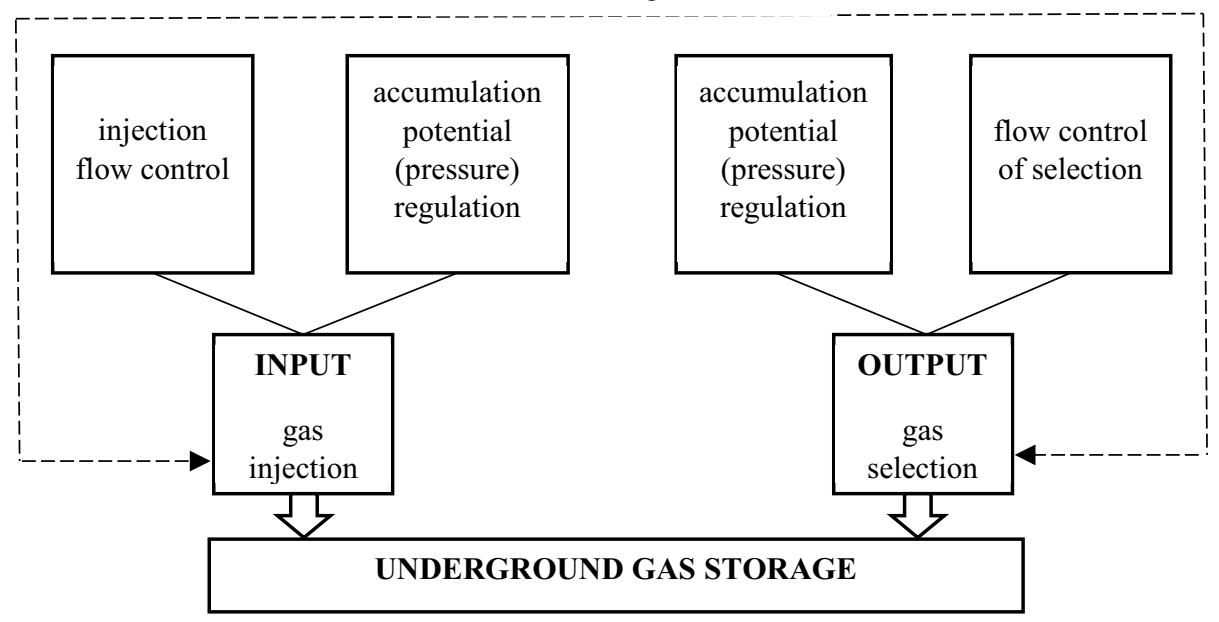

Fig. 2. Regulation of the operation of underground gas storage.

The regulation on the accumulation potential will ensure that the gas pressure change is maintained within the specified limits. If the pressure is higher than the calculated maximum pressure of the reservoir tightness, then the formation of gas griffins is possible.

Consumption / accumulation flow control ensures high stability and control stability by ensuring consumption / accumulation characteristics within the required limits.

Feedback regulation is possible in two modes:

- in the information feedback mode, taking into account the statistics of the inflow volumes of associated petroleum gas, saline water and carbon dioxide;

- in the mode of material feedback on the actual injection of associated petroleum gas, saline water, carbon dioxide and cyclic production of associated petroleum gas and gas condensate.

In the information feedback mode, the information from the primary sensors about the accumulation potential is used to regulate the equipment installed at the entrance to the underground gas storage.

In the material feedback mode, the gas phase is recirculated, the residual gas condensate is displaced, the carbon dioxide is buried, and thereby the functioning of the entire system is maintained.

The presence of two control modes makes the system unstable. How to avoid instability in such sequentially connected control modes? Let us single out two ways of regulating the operation of associated petroleum gas: 
1 - the regulation of the functioning of the system is incomplete, only general trends in the development of events are obtained, and the final decision is made by specialists in accordance with the Regulations;

2 - regulation of the functioning of the system by reducing the depth of feedback, which leads to a decrease in the accuracy of regulation, however, this increases the stability of regulation.

The delay in feedback systems is considered as multicomponent:

- the first component is information-measuring, related to the time of measuring and processing information;

- the second component is the transport delay associated with the real flow;

- the third component is associated with the physicochemical processes occurring in the oil and gas condensate field, which were usually not taken into account and were considered as some external interference or error of the method [3].

Taking into account time delays and the presence of undesirable deviations to ensure the required stability of control, it is preferable to use output control, and the input control is considered as auxiliary to ensure the storage of carbon dioxide with a given volume accuracy [3-5].

The use of an integrated systematic approach to regulation will allow leveling the flow of consumption, optimizing reservoir losses, increasing environmental and industrial safety, and, accordingly, the overall stability of the underground gas storage [6-7].

\section{Discussion and Conclusion}

1. A mathematical model has been developed for the operation of an underground gas storage taking into account the risk of instability in the control of the process of accumulation of hydrocarbon mixtures and carbon dioxide with preliminary recirculation of hydrocarbon mixtures, where the accumulation of carbon dioxide is considered without recirculation.

2. Creation of an underground gas storage facility in a depleted oil and gas condensate field by means of information and mathematical support of the system makes it possible to maintain the specified modes of all objects and installations of underground gas storage in an automatic mode and subsequently use it for carbon dioxide storage.

3. To ensure the stability of the functioning of the objects of accumulation and consumption, it is necessary to calculate the depth of feedback for each component of the component: proportional, integral, differential (PID - regulation).

\section{References}

1. PJSC "Gazprom". Underground gas storage, https://www.gazprom.ru/about/production/underground-storage/

2. J.S. Archer, C.G. Wall, Petroleum Engineering: Principles and Practice (Graham and Trotman Ltd., London, 1986) ISBN 0860106659.

3. Yu. Gromov et al, Systems of automatic control with delay: tutorial (Publishing house of Tamb. state tech. University, Tambov, 2007) ISBN 978-5-8265-0644-8

4. D. Dvinin, E. Nikolaeva, E3S Web of Conferences 03013, 157 (2020) doi:10.1051/e3sconf/202015703015

5. A.M. Bondaruk, S.S. Gots, K.Sh. Yamaletdinova, R.N. Gimaev, Automated quality management systems in technological processes: textbook (OOO "Monograph", Ufa, 2007) ISBN 978-5-94920-086-5 
6. K.Sh. Yamaletdinova, S.S. Gots, Electronic scientific journal "Oil and Gas Business" (2006) http://ogbus.ru/files/ogbus/authors/Yamaletdinova/Yamaletdinova_2.pdf

7. A.A. Yamaletdinova, Study of the peculiarities of the creation of underground gas storage facilities in depleted oil and gas condensate fields: dis. ... Cand. tech. Sciences: 25.00.19 (Ufa, 2015)

8. V. Barkhatov, D. Benz, D. Pletnev, E3S Web of Conferences 13013, 210 (2020) doi:10.1051/e3sconf/202021013013

9. D. Pletnev, E. Nikolaeva, E. Silova, E3S Web of Conferences 13014, 210 (2020) doi:10.1051/e3sconf/202021013014

10. S. Motaghi, A. Mottaghi, D. Pletnev et al, E3S Web of Conferences 14020, 175 (2020) doi:10.1051/e3sconf/202017514020

11. A.A. Yamaletdinova, A.M. Bondaruk, M.R. Gimaev, A.F. Mukhametzyanova, Electronic scientific journal "Oil and Gas Business" (2010) http://ogbus.ru/files/ogbus/eng/authors/Bondaruk/Bondaruk_1.pdf 